\title{
Factors influencing the spread of pertussis in households: a prospective study, Catalonia and Navarre, Spain, 2012 to 2013
}

P Godoy ${ }^{123}$, M García-Cenoz ${ }^{245}$, D Toledo ${ }^{26}$, G Carmona ${ }^{1}$, JA Caylà ${ }^{27}$, M Alsedà ${ }^{13}$, / Àlvarez ${ }^{1}$, I Barrabeig ${ }^{1}$, N Camps ${ }^{1}$, P Plans $^{12}$, M Company ${ }^{1}$, J Castilla ${ }^{24}$, M Sala-Farré ${ }^{1}$, C Muñoz-Almagro ${ }^{289}$, C Rius ${ }^{27}$, Á Domínguez ${ }^{26}$, for the Transmission of Pertussis in Households Working Group ${ }^{10}$

1. Agència de Salut Pública de Catalunya, Barcelona, Spain

2. Ciber de Epidemiología y Salud Pública, CIBERESP, Madrid, Spain

3. Institut de Recerca Biomèdica de Lleida, IRBLleida, Lleida, Spain

4. Instituto de Salud Pública de Navarra, IdiSNA, Pamplona, Spain

5. Universidad Pública de Navarra (UPNA), Navarre, Spain

6. Universitat de Barcelona, Barcelona, Spain

7. Agència de Salut Pública de Barcelona, Barcelona, Spain

8. Hospital de Sant Joan de Dèu, Barcelona, Spain

9. Universitat Internacional de Catalunya, Barcelona, Spain

10. Members of the group are listed at the end of the article

Correspondence: Pere Godoy (pere.godoy@gencat.cat)

Citation style for this article:

Godoy P, García-Cenoz M, Toledo D, Carmona G, Caylà JA, Alsedà M, Àlvarez J, Barrabeig I, Camps N, Plans P, M Company, Castilla J, Sala-Farré M, Muñoz-Almagro C, Rius C, Domínguez À, for the Transmission of Pertussis in Households Working Group. Factors influencing the spread of pertussis in households: a prospective study, Catalonia and Navarre, Spain, 2012 to 2013 . Euro Surveill. 2016;21(45):pii=30393. DOI: http://dx.doi.org/10.2807/1560-7917.ES.2016.21.45.30393

Article submitted on 25 April 2015 / accepted on 28 February 2016 / published on 10 November 2016

We aimed to investigate transmission rates of pertussis in household contacts of cases and factors associated with transmission. A prospective epidemiological study was conducted in 2012 and 2013 to determine the incidence of pertussis among household contacts of reported cases in Catalonia and Navarre, Spain. An epidemiological survey was completed for each case and contact, who were followed for 28 days to determine the source of infection (primary case) and detect the occurrence of secondary cases. Odds ratios (ORs) were used to estimate the effectiveness of vaccination and chemoprophylaxis in preventing new cases, using the formula $(1-O R) \times 100$. For the 688 primary cases, a total of 2,852 contacts were recorded. The household transmission rate was $16.1 \%(459 / 2,852)$ and rose according to the age ( $>18$ years) and lack of immunisation of the primary cases, and also the age (0-18 years), family relationship (siblings and children), lack of vaccination and chemoprophylaxis of contacts. Pertussis vaccine effectiveness in preventing new cases was $65.0 \%$ (95\% confidence interval $(\mathrm{Cl}): 11.6$ to 86.2 ) for full vaccination ( $\geq 4$ doses) and $59.7 \%$ ( $95 \%$ $\mathrm{Cl}:-6.8$ to 84.8 ) for incomplete vaccination ( 4 doses). The effectiveness of chemoprophylaxis was $62.1 \%$ (95\% Cl:40.3 to 75.9). To reduce household transmission, contacts should be investigated to detect further cases and to administer chemoprophylaxis. The current vaccination status of cases and contacts can reduce household transmission.

\section{Introduction}

Pertussis vaccination has led to an important reduction in the incidence of the disease in children in the past 60 years [1]. However, pertussis remains a vaccine-preventable disease that causes a large number of deaths worldwide [2] and has high incidence and hospitalisation rates, even in industrialised countries [3,4].

Studies suggest that the persistence of transmission of the causative agent, Bordetella pertussis, is due to the fact that immunity to $B$. pertussis infection - whether acquired naturally or by vaccination - is not lifelong $[5,6]$. In fact, a second infection in people who have already been infected with $B$. pertussis have been reported [7]. When whole-cell vaccines (wPs) are used, protective antibodies decline by $50 \%$ over a period of 6 to 12 years $[5,8]$. The duration of immunity conferred by acellular vaccines (aPs) - which are used today in most industrialised countries because they are less reactogenic [9] - appears to be shorter than that conferred by WP $[10,11]$. Some studies suggest that aPs induce a suboptimal immune response that is unable to prevent infection, thus providing a plausible explanation for pertussis resurgence [12].

In Spain, the WP against pertussis, combined with diphtheria and tetanus toxoids (DTwP), was commercialised in the 1960s and was administered to infants (agedunder 1 year) in two annual campaigns [13]. In Catalonia and Navarre, the WP was included in 1980 
TABLE 1

Characteristics of primary cases of pertussis with household contacts, Catalonia and Navarre, Spain, 2012-13 $(\mathrm{n}=688)$

\begin{tabular}{|c|c|c|}
\hline Characteristic of primary case & Number & $\%$ \\
\hline \multicolumn{3}{|l|}{ Sex } \\
\hline Male & 325 & 47.2 \\
\hline Female & 363 & 52.8 \\
\hline \multicolumn{3}{|l|}{ Age in years } \\
\hline$<1$ & 151 & 21.9 \\
\hline 1 & 24 & 3.5 \\
\hline $2-3$ & 44 & 6.4 \\
\hline $4-6$ & 76 & 11.0 \\
\hline $7-10$ & 149 & 21.7 \\
\hline $11-18$ & 98 & 14.2 \\
\hline $19-40$ & 76 & 11.0 \\
\hline$>40$ & 70 & 10.2 \\
\hline \multicolumn{3}{|l|}{ Clinical symptoms } \\
\hline Cough lasting > 2 weeks & 644 & 93.6 \\
\hline Paroxysmal cough & 581 & 84.4 \\
\hline Post-tussive vomiting & 276 & 40.1 \\
\hline Inspiratory stridor & 259 & 37.6 \\
\hline Apnoea & 151 & 21.9 \\
\hline Fever & 74 & 10.8 \\
\hline \multicolumn{3}{|c|}{ Laboratory confirmation (PCR and/or culture) } \\
\hline Yes & 504 & 73.3 \\
\hline No & 184 & 26.7 \\
\hline \multicolumn{3}{|l|}{ Hospitalisation } \\
\hline Yes & 105 & $15 \cdot 3$ \\
\hline No & 583 & 84.7 \\
\hline \multicolumn{3}{|l|}{ Vaccination status $^{\mathrm{a}}$} \\
\hline Fully vaccinated & 331 & 48.1 \\
\hline Undervaccinated due to age & 90 & 13.1 \\
\hline Undervaccinated & 15 & 2.2 \\
\hline Unvaccinated & 61 & 8.9 \\
\hline Unvaccinated due to age & 66 & 9.6 \\
\hline Unknown/no answer & 125 & 18.2 \\
\hline
\end{tabular}

a Vaccination status was categorised as fully vaccinated ( $\geq 4$ doses of vaccine), undervaccinated ( 4 doses), unvaccinated (no dose), undervaccinated due to age ( $/ 4$ doses) and unvaccinated due to age (no dose).

in the national childhood immunisation schedule, with four doses at 3, 5, 7 and 18 months of age. In 1998, the vaccination schedule was changed, reducing the age of administration and number of the WP doses at 2, 4 and 6 months of age, and included two doses of the aP (at 18 months and 4-6 years of age). In 2002, five doses of aP - diphtheria, tetanus and acellular pertussis (DTaP)/ combined tetanus, diphtheria and acellular pertussis (Tdap) - were introduced into the childhood immunisation schedule, with the last dose given at the age of 4-6 years, to reduce the side effects of wP vaccination. In Spain, vaccination coverage with pertussis vaccines has been more than $90 \%$ since 1990 [13]. Nevertheless, pertussis incidence increased from less than 1 per
100,000 population in 2003 to 5.3 per 100,000 population in 2013 [13].

Studies of children worldwide hospitalised due to serious outcomes of pertussis have shown that the most frequent source of infection is in the household, due to infection by mothers or other family members (siblings, fathers, grandparents) or caregivers, who presented with symptoms of coughing that were not recognised as being due to pertussis [14-16].

Other studies of community index cases also indicate that $B$. pertussis transmission often occurs in households and that transmission rates in this setting are variable but high, depending on factors related to the pertussis cases and their contacts, such as age, sex or immune status $[17,18]$.

The rate of secondary transmission of $B$. pertussis in Spanish households and the relative importance of family relationships and specific age groups regarding infection is unknown. Similarly, chemoprophylaxis with azithromycin is recommended for post-exposure prophylaxis [19], but its effectiveness, and that of DTwP/DTaP/Tdap vaccination, in preventing transmission in household contacts is also unknown. Such data could be valuable in the assessment of strategies to reduce the number of $B$. pertussis infections, especially in children.

The aim of our study was to investigate the sources of infection of primary cases and rates of secondary transmission of pertussis in contacts of pertussis cases in households and factors associated with transmission in Catalonia and Navarre, Spain.

\section{Methods}

A prospective epidemiological study was conducted in 2012 and 2013 on the incidence of pertussis among household contacts of pertussis cases who were reported to the notifiable diseases systems of Catalonia and Navarre, which together have a population of 8.2 million [20].

Index cases (defined below) were reported to public health professionals from the epidemiological surveillance units of the Department of Health of Catalonia, the Public Health Agency of Barcelona and the Public Health Institute of Navarre. Each case notified was considered an index case. To be included in the study, an individual had to meet the criteria for a confirmed case (see below) and have household contacts who could be identified.

For each index case detected, an epidemiological survey of the study variables (outlined below) was completed and household contacts were identified. Each case was asked about exposure to a person with pertussis, symptoms, doses of pertussis vaccine received (registered in an official document or medical history) and preventive measures adopted (vaccination or 
Characteristics of household contacts of primary cases of pertussis, Catalonia and Navarre, Spain, 2012-13 $(\mathrm{n}=2,852)$

\begin{tabular}{|c|c|c|}
\hline Characteristic of household contact & Number & $\%$ \\
\hline \multicolumn{3}{|l|}{ Sex } \\
\hline Male & 1,340 & 47.0 \\
\hline Female & 1,512 & 53.0 \\
\hline \multicolumn{3}{|l|}{ Age in years } \\
\hline$\ll 1$ & 150 & 5.3 \\
\hline 1 & 58 & 2.0 \\
\hline $2-3$ & 132 & 4.6 \\
\hline $4-6$ & 200 & 7.0 \\
\hline 7-10 & 221 & 7.7 \\
\hline $11-18$ & 209 & 7.3 \\
\hline $19-40$ & 967 & 33.9 \\
\hline$>40$ & 915 & 32.1 \\
\hline \multicolumn{3}{|l|}{ Household contacts } \\
\hline Cohabitant & 2,034 & 71.3 \\
\hline Non-cohabitant & 818 & 28.7 \\
\hline \multicolumn{3}{|l|}{ Relationship to primary case } \\
\hline Mother & 556 & 19.5 \\
\hline Father & 510 & 17.9 \\
\hline Sibling & 518 & 18.2 \\
\hline Grandparent & 330 & 11.6 \\
\hline Child & 139 & 4.9 \\
\hline Partner & 100 & 3.5 \\
\hline Other $^{\mathrm{a}}$ & 699 & 24.5 \\
\hline \multicolumn{3}{|l|}{ Number of contacts in the household } \\
\hline$\leq 2$ & 226 & 7.9 \\
\hline $3-4$ & 1,133 & 29.7 \\
\hline$>4$ & 1,493 & 52.3 \\
\hline \multicolumn{3}{|l|}{ Vaccination status $(\leq 18 \text { years })^{b}$} \\
\hline Fully vaccinated & 581 & 64.4 \\
\hline Undervaccinated due to age & 94 & 10.4 \\
\hline Undervaccinated & 27 & 3.0 \\
\hline Unvaccinated & 49 & 5.4 \\
\hline Unvaccinated due to age & 53 & 5.9 \\
\hline Unknown/no answer & 97 & 10.8 \\
\hline \multicolumn{3}{|l|}{ Received chemoprophylaxis ${ }^{c}$} \\
\hline Yes & 2,284 & 80.1 \\
\hline No & 406 & 14.2 \\
\hline Unknown & 162 & 5.7 \\
\hline
\end{tabular}

a Caregiver, family friend or neighbour.

b Vaccination status was categorised as fully vaccinated ( $\geq 4$ doses of vaccine), undervaccinated ( 4 doses), unvaccinated (no dose), undervaccinated due to age ( 4 doses) and unvaccinated due to age (no dose).

${ }^{c}$ Azithromycin was used.

chemoprophylaxis). As the transmission period of the disease may be as long as 21 days [21] and the incubation period in a new case seven days [22], cases and contacts were followed for 28 days to determine the source of infection (primary case), and the appearance of secondary cases.
Two samples were taken, using appropriate swabs (Dacron or Rayon for PCR) and cotton for cultures), from the posterior nasopharynx of each case and contact with pertussis-compatible symptoms for determining presence of $B$. pertussis by culture or PCR. Swabs for culture were transported in a suitable medium to ensure viability of the bacteria and swabs for PCR were resuspended in $200 \mu \mathrm{L}$ saline solution. B. pertussis DNA was detected using real-time PCR amplification of the insertion sequences Bordetella IS481 [23]. Human RnaseP gene was used to check sample quality and detection of inhibitors of PCR reaction.

\section{Definitions}

An index case was defined as the first reported pertussis case who generated the study of pertussis in a particular household.

A confirmed case was defined as a person presenting clinically with a cough, together with microbiological confirmation (isolation of $B$. pertussis in culture or positive PCR test from nasopharyngeal swabs) or a person who fulfilled the clinical definition (cough for more than two weeks and at least one of the following: paroxysmal cough, inspiratory stridor, post-tussive vomiting or apnoea) and who was also epidemiologically linked to a confirmed case.

A primary case was defined as the first confirmed case of pertussis in a household to develop symptoms.

A coprimary case was defined a confirmed case of pertussis with symptoms appearing between o and 6 days after those of the primary case had started.

A secondary case was a confirmed case in whom symptoms began between 7 and 28 days after those of the primary case.

After completion of the survey and laboratory tests, each index case and household contact was classified as a healthy contact, primary case or secondary case (confirmed microbiologically or by epidemiological link).

Household contacts were defined as all residents of the household of the primary case (cohabitants) or persons who had had contact with the primary case formore than 2 hours (to exclude sporadic contact) in the same dwelling during the transmission period of the disease (non-cohabitants) in order to detect cases among relatives and caregivers who were not household cohabitants but could have a relevant role in the epidemiological chain. We choose 2 hours to eliminate sporadic contact (with less than 2 hours of contact). The transmission period of the disease was defined as the period of 21 days from the onset of symptoms in the primary case or five days from the onset of treatment of the primary case. 
TABLE 3

Incidence of pertussis in household contacts by characteristic of primary cases $(n=2,852)$

\begin{tabular}{|c|c|c|c|c|}
\hline \multirow{2}{*}{$\begin{array}{l}\text { Characteristic of } \\
\text { primary case }\end{array}$} & \multicolumn{2}{|c|}{$\begin{array}{l}\text { Incidence of pertussis } \\
\text { among contacts }\end{array}$} & \multirow[t]{2}{*}{ Odds ratio } & \multirow[t]{2}{*}{$95 \% \mathrm{Cl}$} \\
\hline & $\%$ & $\mathrm{n} /$ total & & \\
\hline \multicolumn{5}{|l|}{ Sex } \\
\hline Female & 16.0 & $245 / 1,528$ & 1.0 & 0.8 to 1.2 \\
\hline Male & 16.2 & $214 / 1,324$ & \multicolumn{2}{|c|}{ Reference } \\
\hline \multicolumn{5}{|l|}{ Age in years } \\
\hline$\langle 1$ & 8.9 & $60 / 671$ & \multicolumn{2}{|c|}{ Reference } \\
\hline 1 & 9.6 & $10 / 104$ & 1.1 & 0.5 to 2.2 \\
\hline $2-3$ & 10.9 & $25 / 229$ & 1.2 & 0.7 to 2.0 \\
\hline $4-6$ & 14.4 & $47 / 326$ & 1.7 & 1.1 to 2.6 \\
\hline $7-10$ & 15.3 & $91 / 595$ & 1.8 & 1.3 to 2.6 \\
\hline $11-18$ & 14.9 & $54 / 363$ & 1.8 & 1.2 to 2.6 \\
\hline $19-40$ & 31.0 & $90 / 290$ & 4.6 & 3.2 to 6.6 \\
\hline$>40$ & 29.9 & $82 / 274$ & 4.3 & 3.0 to 6.3 \\
\hline \multicolumn{5}{|c|}{ Microbiological confirmation (PCR and/or culture) } \\
\hline Yes & 10.7 & $219 / 2,055$ & \multicolumn{2}{|c|}{ Reference } \\
\hline No & 27.0 & $61 / 226$ & 3.1 & 2.2 to 4.3 \\
\hline Unknown & 30.8 & $158 / 513$ & 3.7 & 2.9 to 4.7 \\
\hline \multicolumn{5}{|l|}{ Hospitalisation } \\
\hline Yes & 10.0 & $48 / 479$ & 0.6 & 0.4 to 0.8 \\
\hline No & 17.4 & $403 / 2,312$ & \multicolumn{2}{|c|}{ Reference } \\
\hline \multicolumn{5}{|l|}{ Number of contacts } \\
\hline$\leq 2$ & 19.5 & $44 / 226$ & \multicolumn{2}{|c|}{ Reference } \\
\hline $3-4$ & 16.0 & $181 / 1,133$ & 0.8 & 0.6 to 1.1 \\
\hline$>4$ & 15.7 & $234 / 1,493$ & 0.8 & 0.6 to 1.1 \\
\hline \multicolumn{5}{|l|}{ Vaccination status $^{a}$} \\
\hline Fully vaccinated & 14.1 & $188 / 1,331$ & \multicolumn{2}{|c|}{ Reference } \\
\hline Undervaccinated/ Unvaccinated/Unknown & 17.8 & $271 / 1,521$ & 1.3 & 1.1 to 1.6 \\
\hline
\end{tabular}

$\mathrm{Cl}$ : confidence interval.

a Vaccination status was categorised as fully vaccinated ( $\geq 4$ doses of vaccine), undervaccinated ( 4 doses) or unvaccinated (no dose).

\section{Study variables}

Information on the following sets of variables was obtained from a face-to-face questionnaire and official records for each pertussis case and each household contact.

Demographic variables: sex, age, number of persons in a household (cohabitant or non-cohabitant) and the relationship between the household members. For contacts, the relationship with the primary case (e.g. mother, father, sibling, grandparent, child, partner, other) was recorded.

Clinical variables: date of onset of first symptom, cough lasting 2 or more weeks, number of days of persistent cough, and presence/absence of paroxysmal coughing, post-tussive vomiting, apnoea, fever, pneumonia, seizures, encephalopathy, hospitalisation.

Laboratory results: type of sample, result of culture and PCR.
Preventive measures: for study participants - all cases of pertussis (all ages) and household contacts (aged $\leq 18$ years) - who had received any dose of pertussis vaccine, the number and date of administration of doses were recorded. The cut-off of 18 years was chosen because few contacts aged more than 18 years had records of their vaccinations. Vaccination status was categorised as fully vaccinated ( $\geq 4$ doses of vaccine), undervaccinated ( 4 doses), unvaccinated (no dose), undervaccinated due to age ( $\$ 4$ doses) and unvaccinated due to age (no dose).

Chemoprophylaxis was defined as completion of antibiotic treatment (azithromycin) in a healthy contact (all ages) initiated after symptom onset of the primary case.

\section{Sample size}

Given that the annual median number of new cases in Catalonia and Navarre was 203 [24] and the study 


\section{TABLE 4}

Incidence of pertussis in household contacts by characteristic, Catalonia and Navarre, Spain, 2012-13 $(\mathrm{n}=2,852)$

\begin{tabular}{|c|c|c|c|c|}
\hline \multirow{2}{*}{$\begin{array}{l}\text { Characteristic of } \\
\text { household contact }\end{array}$} & \multicolumn{2}{|c|}{$\begin{array}{l}\text { Incidence of pertussis } \\
\text { among contacts }\end{array}$} & \multirow{2}{*}{$\begin{array}{l}\text { Odds } \\
\text { ratio }\end{array}$} & \multirow{2}{*}{$95 \% \mathrm{Cl}$} \\
\hline & $\%$ & $\mathrm{n} /$ total & & \\
\hline \multicolumn{5}{|l|}{ Sex } \\
\hline Female & 15.9 & $241 / 1,512$ & 1.0 & \begin{tabular}{|c|}
0.8 to \\
1.2 \\
\end{tabular} \\
\hline Male & 16.3 & $218 / 1,340$ & \multicolumn{2}{|c|}{ Reference } \\
\hline \multicolumn{5}{|l|}{ Age in years } \\
\hline$\ll 1$ & 69.3 & $104 / 150$ & 24.6 & $\begin{array}{c}16.2 \text { to } \\
37.4\end{array}$ \\
\hline 1 & 44.8 & $26 / 58$ & 8.8 & $\begin{array}{c}5.0 \text { to } \\
15.6 \\
\end{array}$ \\
\hline $2-3$ & 25.0 & $33 / 132$ & 3.6 & $\begin{array}{c}2.3 \text { to } \\
5.7\end{array}$ \\
\hline $4-6$ & 21.5 & $43 / 200$ & 3.0 & $\begin{array}{c}2.0 \text { to } \\
4.5\end{array}$ \\
\hline $7-10$ & $19 \cdot 5$ & $43 / 221$ & 2.6 & $\begin{array}{c}1.7 \text { to } \\
3.9\end{array}$ \\
\hline $11-18$ & 19.1 & $40 / 209$ & 2.6 & $\begin{array}{c}1.7 \text { to } \\
3.9\end{array}$ \\
\hline $19-40$ & 9.6 & $93 / 967$ & 1.2 & $\begin{array}{c}0.8 \text { to } \\
1.6\end{array}$ \\
\hline$>40$ & 8.4 & $77 / 915$ & \multicolumn{2}{|c|}{ Reference } \\
\hline \multicolumn{5}{|l|}{ Household contacts } \\
\hline Cohabitants & 16.5 & $336 / 2034$ & 1.1 & \begin{tabular}{|c|}
0.9 to \\
1.4 \\
\end{tabular} \\
\hline Non-cohabitants & 15.0 & $123 / 818$ & \multicolumn{2}{|c|}{ Reference } \\
\hline \multicolumn{5}{|c|}{ Relationship with primary case } \\
\hline Mother & 8.3 & $46 / 556$ & 1.8 & $\begin{array}{c}1.0 \text { to } \\
3.4\end{array}$ \\
\hline Father & 8.8 & $45 / 510$ & 2.0 & $\begin{array}{c}1.1 \text { to } \\
3.7\end{array}$ \\
\hline Sibling & $25 \cdot 7$ & $133 / 518$ & 7.2 & $\begin{array}{l}4.2 \text { to } \\
12.6\end{array}$ \\
\hline Grandparent & 4.5 & $15 / 330$ & \multicolumn{2}{|c|}{ Reference } \\
\hline Child & 61.2 & $85 / 139$ & 33.0 & \begin{tabular}{|c|}
17.7 to \\
61.5 \\
\end{tabular} \\
\hline Partner & 16.0 & $16 / 100$ & 4.0 & $\begin{array}{c}1.9 \text { to } \\
8.4 \\
\end{array}$ \\
\hline Other ${ }^{\mathrm{a}}$ & 17.0 & $119 / 699$ & $4 \cdot 3$ & $\begin{array}{c}2.5 \text { to } \\
7.5\end{array}$ \\
\hline \multicolumn{5}{|c|}{ Vaccination status $^{\mathrm{b}}$ ( $\leq 18$ years) } \\
\hline Fully vaccinated & 23.8 & $138 / 581$ & 0.11 & $\begin{array}{c}0.07 \text { to } \\
0.17 \\
\end{array}$ \\
\hline Undervaccinated & 52.9 & $64 / 121$ & 0.38 & \begin{tabular}{|c|}
0.22 to \\
0.68 \\
\end{tabular} \\
\hline Unvaccinated & 74.5 & $76 / 102$ & \multicolumn{2}{|c|}{ Reference } \\
\hline \multicolumn{5}{|c|}{ Received chemoprophylaxis ${ }^{c}$} \\
\hline Yes & 9.9 & $226 / 2,284$ & 0.47 & \begin{tabular}{|c|}
0.35 to \\
0.62
\end{tabular} \\
\hline No & 19.0 & $77 / 406$ & \multicolumn{2}{|c|}{ Reference } \\
\hline
\end{tabular}

$\mathrm{Cl}$ : confidence interval.

a Caregiver, family friend or neighbour.

b Vaccination status was categorised as fully vaccinated ( $\geq 4$ doses of vaccine), undervaccinated ( 4 doses) or unvaccinated (no dose).

c Azithromycin was used. period was 2 years, we expected to register 406 new cases during the study. Taking a mean of three household contacts (excluding the index case), we expected to register 1,218 household contacts. The median size of families in Spain is 2.5 members [25]; however, as other contacts in households, such as caregivers, were included, we decided to use a mean of three.

The rate of transmission in households, assuming an expected level of $10 \%$ [21], was estimated to a precision of $\pm 1.7 \%$.

\section{Data analysis}

Primary cases and contacts were described using percentages with their 95\% confidence intervals (Cls) for qualitative variables, and means and standard deviation (SD) for quantitative variables.

The rate of transmission with its $95 \% \mathrm{Cl}$ was calculated using the formula:

\section{Formula 1}

Secondary transmission rate $=($ Cases detected among the household contacts of primary cases/Total number of household contacts) $\times 100$

Primary cases were not included in the numerator or the denominator.

The risk of transmission was studied according to the characteristics of primary cases and their household contacts using the chi-squared test for qualitative variables and the ANOVA or Kruskall tests for quantitative variables, with a level of significance of $p<0.05$. The strength of an association was calculated using odds ratios (ORs) and their 95\% Cls.

The vaccine effectiveness (only in household contacts aged 18 years or under) and chemoprophylaxis (in all household contacts) was studied using the formula: Effectiveness $=(1-$ OR $) \times 100$. The estimated ORs were adjusted using an unconditional logistic regression model produced by eliminating variables using stepwise regression in which predictive variables were carried out by the automatic backward method starting with all candidate variables and eliminating variables from $p<0.2$.

The variables evaluated in the models were vaccination status, use of chemoprophylaxis, age, sex and family relationship of the contacts, in addition to the sex, age and vaccination status of the primary case.

\section{Ethical aspects}

The study was approved by the Ethics Committee of the Hospital Sant Joan de Deu (code: PIC-79-11). All contacts and family members were informed about the study and gave their consent to participate. 


\section{TABLE 5}

Multivariate analysis of the effectiveness of pertussis vaccination and chemoprophylaxis of household contacts in reducing household transmission, Catalonia and Navarre, Spain, 2012-13

\begin{tabular}{|l|c|c|c|}
\hline $\begin{array}{l}\text { Characteristic of } \\
\text { household contact }\end{array}$ & $\begin{array}{c}\text { Adjusted } \\
\text { odds ratio }\end{array}$ & $95 \% \mathrm{Cl}$ & $\mathrm{p}$ value \\
\hline Vaccination status ${ }^{\mathrm{b}}(\leq 18$ years) \\
\hline Fully vaccinated & 0.350 & $\begin{array}{c}0.138 \text { to } \\
0.884\end{array}$ & 0.026 \\
\hline Undervaccinated & 0.403 & $\begin{array}{c}0.152 \text { to } \\
1.068\end{array}$ & 0.067 \\
\hline Unvaccinated & \multicolumn{4}{|c|}{ Reference } & - \\
\hline Received chemoprophylaxis ${ }^{\mathrm{c}}$ & \multicolumn{4}{|c|}{$\begin{array}{c}0.241 \text { to } \\
0.597\end{array}$} & 0.001 \\
\hline Yes & 0.379 & - \\
\hline No & \multicolumn{4}{|c|}{ Reference } & - \\
\hline
\end{tabular}

$\mathrm{Cl}$ : confidence interval.

a Adjusted by age of contacts, sex of contacts, relationship with primary case, sex of primary case, age of primary case and pertussis vaccination status of primary case.

${ }^{b}$ Vaccination status was categorised as fully vaccinated ( $\geq 4$ doses of vaccine), undervaccinated ( 44 doses) or unvaccinated (no dose).

Azithromycin was used.

\section{Results}

We studied 688 index cases, of whom $76.2 \%$ (524/688) were the primary cases in the household. The remainder (164/688) were secondary cases (household contacts). Thus the 688 primary cases studied (the first cases who became symptomatic in a household) comprised 524 index cases and 164 household contacts who were identified as primary cases once the study of the household was complete.

Of these 688 confirmed primary cases, $52.8 \%$ were female, $21.9 \%$ were agedunder 1 year, $42.6 \% \quad 1-10$ years, $14.2 \% \quad 11-18$ years and $21.2 \%$ more than 18 years. Primary cases had the following symptoms: cough lasting more than 2 weeks (93.6\%), paroxysmal cough $(84.4 \%)$, post-tussive vomiting (40.1\%), inspiratory stridor (37.6\%), apnoea (21.9\%) and fever (10.8\%) (Table 1). The frequency of symptoms experienced by primary cases aged more than 18 years was slightly different: coughlasting more than 2 weeks (98.6\%), paroxysmal cough (84.4\%), post-tussive vomiting (23.1\%), inspiratory stridor (30.6\%), apnoea (20.4\%) and fever $(6.8 \%)$. Of the 688 primary cases, $15.3 \%$ were hospitalised, including $63.6 \%(96 / 151)$ of those aged under 1 year.

Laboratory confirmation (PCR and/or culture) was obtained for $73.3 \%(n=504)$ of the primary cases and by epidemiological link in $26.7 \%(n=184) ; 48.1 \%$ of cases were fully vaccinated (they had received $\geq 4$ doses of vaccine), $13.1 \%$ were undervaccinated due to age, $2.2 \%$ were simply undervaccinated, $8.9 \%$ had received no vaccine dose and $9.6 \%$ were unvaccinated due to age (Table 1 ).
A total of 2,852 household contacts of the 688 primary cases were recorded, of whom $52.8 \%$ were female, $66.0 \%$ were older than 18 years, $7.3 \%$ were aged $11-18$ years and $26.6 \%$ were under 11 years. About $71 \%$ of the contacts were cohabitants, i.e. they lived in the same household as the primary case. The most common family relationships among the contacts were being a mother $(19.5 \%)$, father $(17.9 \%)$ or sibling $(18.2 \%)$ of the primary case. Some $64 \%$ of contacts aged $\leq 18$ years were fully vaccinated, $13 \%$ were undervaccinated and $11 \%$ were unvaccinated (Table 2 ).

The household transmission rate (incidence of pertussis among household contacts) was $16.1 \%$ (459/2,852) and was slightly higher when the primary case was male $(16.2 \%)$, but this difference was not statistically significant. Compared with data from primary cases aged under 1 year, the household transmission rate was higher when the primary case was aged $4-6$ years (OR: 1.7; $95 \% \mathrm{Cl}: 1.1$ to 2.6 ), 7-10 years (OR: $1.8 ; 95 \% \mathrm{Cl}: 1.3$ to 2.6 ), $11-18$ years (OR: $1.8 ; 95 \% \mathrm{Cl}: 1.2$ to 2.6 ), $19-40$ years (OR: $4.6 ; 95 \% \mathrm{Cl}: 3.2$ to 6.6 ) and older than 40 years $(\mathrm{OR}=4.3 ; 95 \% \mathrm{Cl}: 3.0$ to 6.3$)$. It was also higher when the primary case was undervaccinated, unvaccinated or of unknown vaccination status (OR:1.3; 95\% $\mathrm{Cl}: 1.1$ to 1.6$)$, when compared with primary cases who were fully vaccinated (Table 3 ).

There was no statistically significant difference between the transmission rate in households with 2 or fewer contacts $(19.5 \%), 3-4$ contacts $(16.0 \%)$ ormore than 4 contacts $(15.7 \%)$ ( $p>0.05)$ (Table 3$)$.

When looking at the transmission rate assessed according to variables of household contacts, the rate was slightly higher in male contacts (16.3\%) than in female $(15.9 \%)$, but this difference was not statistically significant. The rate was considerably higher in contacts aged under 1 year (OR: $24.6 ; 95 \% \mathrm{Cl}: 16.2$ to 37.4 ), 1 year (OR: 8.8; 95\% Cl: 5.0 to 15.6 ), $2-3$ years (OR:3.6; 95\% Cl: 2.3 to 5.7 ), $4-6$ years (OR:3.0; $95 \% \mathrm{Cl}: 2.0$ to 4.5), 7-10 years (OR: $2.6 ; 95 \% \mathrm{Cl}: 1.7$ to 3.9 ) and $11-18$ years $(\mathrm{OR}=2.6 ; 95 \% \mathrm{Cl}: 1.7-3.9)$, compared with those aged more than 40 years (Table 4 ).

No difference in transmission rate was observed between contacts who were cohabitants and those who were non-cohabitants (with exposure formore than 2 hours in the household of the primary case). However, the transmission rate was higher in siblings (OR: 7.2; 95\% Cl: 4.2 to 12.6 ) and children (OR: $33.0 ; 95 \% \mathrm{Cl}: 17.7$ to 61.5 ) of primary cases (Table 4 ).

Vaccine effectiveness in household contact aged $\leq 18$ years was $89 \%$ ( $95 \% \mathrm{Cl}: 83$ to 93 ) in reducing transmission in contacts vaccinated with 4 or fewer doses and $62 \%$ ( $95 \% \mathrm{Cl}: 32$ to 78 ) in undervaccinated contacts.

Chemoprophylaxis in all contacts had an effectiveness of $53 \%$ (95\% Cl: 38 to 65 ) in avoiding new cases. 
In the multivariate analysis, the effect of vaccination and chemoprophylaxis for contacts in avoiding new cases was still seen. Vaccine effectiveness in reducing transmission in contacts aged $\leq 18$ years was $65.0 \%$ (95\% Cl: 11.6 to 86.2 ) for full vaccination and $59.7 \%$ ( $95 \% \mathrm{Cl}:-6.8$ to $84.8 \%$ ) for undervaccinated contacts. The adjusted effectiveness of chemoprophylaxis, based on adjusted ORs (Table 5), in all contacts was $62.1 \%$ (95\% Cl: 40.3 to 75.9 ).

\section{Discussion}

The results of this study show that the rate of household transmission of pertussis in Spain in 2012 and 2013 was high, especially in contacts agedunder 18 years, siblings and children of a primary case, unvaccinated contacts and those who had not received chemoprophylaxis.

Household transmission of pertussis is known to be related to the characteristics of primary cases and their contacts [26]. We found increased transmission in households of primary cases aged 18-40 years and those older than 40 years. In the age group 18-40 years, this could be due to closer contact between children and the primary case, especially mothers, due to dependence $[15,27]$. For primary cases aged more than 40 years, the increased rate of transmission might be due to atypical clinical presentation, possibly resulting in important diagnostic delays and therefore more opportunities for transmission $[16,27]$. Lack of vaccination or undervaccination of the primary case also resulted in an increased transmission rate, as observed in other studies $[28,29]$, showing that although full vaccination may not avoid the disease for some cases, it may reduce transmission from the primary case.

In our study, $35.4 \%$ of primary cases were adolescents (11-18 year-olds) or adults (>18 years). Other studies also suggest that adolescents and adults are an important reservoir of the pathogen and source of transmission to children, who are more vulnerable to infection and susceptible to serious complications [28]. In a report published in 1995, Wirsing von König et al. studied pertussis cases in 122 homes in 1995 in an area of Germany with very low vaccination coverage and estimated that adults were the source of infection in $15 \%$ of cases [18]. Later, Baptista et al. studied pertussis cases in 57 homes in Recife, Brazil, in 2003 and found that adults were the primary source of infection in $21.1 \%$ of cases [21,30]. Deen et al. studied 39 homes and 255 exposed persons in Los Angeles, United States, in 1995: in 53\% of households, the primary case was aged older than 12 years [31]. Sala-Farré et al. investigated 59 clusters in an area of Barcelona in 2011 and found that the most frequent primary cases were children aged $5-9$ years (29\%), followed by adults aged 30-39 years (22\%) [32].

In Catalan children in 2001 hospitalised due to severe symptoms of pertussis [33], the source of infection was determined for $63 \%$ of cases and for $44.6 \%$ of those whose infection source was determined, the source was an adolescent or adult. It is recognised that adolescents and adults may act as a source of infection of children [14], but in these age groups the disease is often not diagnosed and is generally under-detected [34]. In a study in Massachusetts, United States, in 1981 to 1991 Marchant et al. [23,35] found an increase in the incidence of confirmed cases in adolescents aged 11-19 years from 3 per 100,000 population to 12.9 per 100,000 population, after facilitating general practitioners' access to serological diagnoses. In another study in Catalonia in 2013, the prevalence of $B$. pertussis infection in the previous 12 months was $1.8 \%$ in women of childbearing age (15-49 years), which suggests there is potentially a high risk for newborns [36]. Studies in various countries that included children hospitalised due to severe disease have shown that the most frequent sources of infection were mothers or other family members (fathers, teenage siblings and grandparents) who presented with coughing that had not been recognised as due to pertussis $[16,21,27,37]$.

The rate of familial transmission from primary cases has been estimated in some studies. In the 1990s, Wirsing von König et al. found a high transmission rate of $26.7 \%$ in adult household contacts in an area of Germany with very low vaccination coverage [18] and in 2003 , in Brazil, Baptista et al. found a rate of secondary transmission of $12.6 \%$ in adult household contacts [30].

In our study, we observed no differences between the number of contacts and transmission rate in the household. Similarly, in the study of Wirsing von König et al. the overall attack rate in adult contacts was independent of the family size [18] but an ecological study from 2009-13 in Minnesota, United States, reported a greater rate of pertussis in counties with a larger average household size [38].

The main characteristics of contacts with an increased transmission rate in our study were being $0-18$ years of age, the sibling or child of a primary case, not vaccinated or undervaccinated and not receiving chemoprophylaxis. In terms of age, we observed a reduction of transmission in the 11-18-year age group and in adults compared with that of the other age groups. This may be due to vaccination with WP, as suggested by the World Health Organization (WHO) position paper on pertussis vaccines [9]. Reduced transmission in household adults was observed in the study of Baptista et al. in Recife, Brazil, in 2003 . Some $87 \%$ of adults exposed to pertussis in the household did not acquire the disease: this was attributed to naturally acquired immunity [30]. In Catalonia and Navarre, five doses of aP were introduced into the official vaccination schedule in 2002 and therefore it may be assumed that most children aged under 11 years in our study were vaccinated with the aP. Specific responses to these changes, such as an adolescent booster dose (after the dose given at 
age 4-6 years) and additional booster doses in adults, may be required.

Pertussis has re-emerged as an important public health concern in Europe since the current aP replaced the older WP. Warfel et al. showed that non-human primates receiving aP were protected from severe symptoms but not infection, and readily transmitted $B$. pertussis to contacts [39]. Key differences in T-cell memory suggest that aP vaccination induces a suboptimal immune response that is unable to prevent infection and provide a plausible explanation for pertussis resurgence [39]. Various studies suggest that attaining herd immunity will require the development of improved vaccination strategies that prevent $B$. pertussis colonisation and transmission $[34,39,40]$.

The increased risk of transmission to siblings of primary cases seen in our study has also been observed by others $[27,41]$. The adjusted vaccine effectiveness of $65 \%$ in avoiding new cases in household contacts aged $\leq 18$ years is similar to or higher than that observed in other studies [42]. Sheridan et al. found an effectiveness of $53 \%$ or $64 \%$, depending on the method of calculation used [43]. However, a position paper by the World Health Organization (WHO) [9] and a systematic review published in the Cochrane database [44] suggest the effectiveness is somewhat higher: $84-85 \%$ in preventing typical whooping cough and $71-78 \%$ in preventing mild pertussis disease. The effectiveness of chemoprophylaxis with azithromycin in our study in preventing transmission was high (62.1\%), suggesting that the detection of pertussis cases, analysing their contacts, and chemoprophylaxis may reduce household transmission, as has been suggested by others [21]. The evidence for the effectiveness of chemoprophylaxis in reducing transmission in household contacts is weak and based on expert opinion $[45,46]$. The results of our study and a recent cost-utility analysis [47] support the use of chemoprophylaxis in household contacts.

Our study has some limitations. First, it was based on notified cases of pertussis, which are known to be underdetected [35]. However, on the basis of the selection of study cases (confirmed cases with household contacts), an active search for contacts with pertussis symptoms was carried out using the survey and the taking of samples from all symptomatic household contacts of primary cases. To ensure all cases were detected, contacts were followed for 28 days from confirmation of the index case. Nevertheless, there may have been transmission due to asymptomatic cases beyond the 28 days of follow-up and the incidence of pertussis may be underestimated. We may not have identified individuals in a household who had recently been infected but may not have reported any specific symptoms. Thus, what is measured and presented in this study is the effectiveness of preventing clinically notifiable disease and not the prevention of infection. Second, vaccination status was collected by documented evidence of vaccination in an official document or medical records: some patients could have been classified as unvaccinated due to vaccination not being recorded, but if such a mistake applies equally to household contacts who remain healthy and those who become pertussis cases, it should not alter the estimated vaccine effectiveness. Third, chemoprophylaxis was recommended to all contacts without symptoms after detection of the index case. Some contacts who received chemoprophylaxis might appear as cases due to continuous exposure to other cases of pertussis in the household and, therefore, the effectiveness of chemoprophylaxis may be underestimated. However, our estimate was obtained after having followed routine pertussis control practices and may be a good estimate of the expected effectiveness when chemoprophylaxis is prescribed by public health services.

In conclusion, the results of our study suggest that in order to reduce household transmission household contacts should be investigated to detect secondary cases and administer chemoprophylaxis rapidly. All contacts who have not received the correct number of doses of pertussis vaccine according to the vaccination schedule should be vaccinated, in addition to receiving chemoprophylaxis. The incidence rate was lower in fully vaccinated individuals and therefore cases could be avoided in the future, although not the immediate future, as pertussis vaccine is not effective as postexposure prophylaxis [47]. The previous pertussis vaccination status of cases and contacts is important in reducing the rate of household transmission. The administration of an additional dose of vaccine in adolescents and adults (especially those in contact with children) could also help to reduce the transmission rate [42]. Nevertheless, there is now increasing evidence that protection following booster doses of aP vaccines wanes faster in individuals primed with $\mathrm{aP}$ rather than with wP vaccines $[9,34,39,40]$. Such vaccination programmes have an impact in directly targeted populations, but there is as yet no substantial evidence that they have had an important impact on severe pertussis in infants. Thus, WHO recommends that national programmes consider vaccinating pregnant women with one dose of Tdap (in the second or third trimester and preferably at least 15 days before the end of the pregnancy) in addition to routine primary infant pertussis vaccination [9]. Ongoing surveillance of pertussis will be critical to monitor the changing epidemiology as the first 'all-aP'-primed cohorts reach adulthood.

Transmission of Pertussis in Households Working Group

The members of the Transmission of Pertussis in Households Working Group (PI11/02557) are as follows: Miquel Alsedà (MA), Josep Alvarez (JA), Cesar Arias (CA), Irene Barrabeig (IB), Neus Camps (NC), Glòria Carmona (GC), Mónica Carol (MCar), Maria Company (MC), Joaquim Ferràs (JF), Glòria Ferrús (GF), Mireia Jané (MJ), Sofia Minguell (SM), Raquel Rodríguez (RR), María-Rosa Sala (M-RS), Roser Torra (RT) (Agència de 
Salut Pública de Catalunya), Pere Godoy (PG), Pedro Plans (PP) (Agència de Salut Pública de Catalunya and CIBERESP), Inma Crespo (IC), Diana Toledo (DT), Àngela Domínguez (AD) and Rubén Solano (RS) (Universitat de Barcelona and CIBERESP), Joan Caylà (JCay), Sara Lafuente (SL) and Cristina Rius (CR) (Agència de Salut Pública de Barcelona and CIBERSP), Manuel GarcíaCenoz (MG-C), Rosana Burgui (RB), Jesús Castilla (JCast) (Instituto de Salud Pública de Navarra, Pamplona and CIBERESP), Ana Valero-Rello (AV-R), Iolanda Jordan (IJ) and Carmen Muñoz-Almagro CM-A) (Hospital de Sant Joan de Dèu, Barcelona).

\section{Acknowledgements}

This study was supported by the Ministry of Science and Innovation, Institute of Health Carlos III (Project PI11/02557) and Fondo Europeo de Desarrollo Regional (FEDER-Una manera de hacer Europa).

\section{Conflict of interest}

None declared.

\section{Authors' contributions}

PG, MG-C, GC, MA, JA, IB, JCast, PP, AD designed the study protocol and participated in the research call for funding for the study. PG, MG-C, GC, DT, PP, CM-A organised the logistics, sought approval from the bioethics committee and obtained informed consent from patients. PG, MG-C, DT, MA, JA, IB, NC, MC, M-RS-F, CR, MCarol, JF, GF, MJ, SM, RR, RT, IC, $\mathrm{RS}, \mathrm{SL}, \mathrm{RB}$ participated in the detection of index cases, recording and tracking of contacts, gathering epidemiological information and taking clinical samples and sending samples to the laboratory. CM-A, IJ, AV-R conducted and performed the microbiological analyses of clinical samples and sent the results to epidemiologists. PG, MG-C, DT, GC, JCay designed the databases of index cases and contacts and conducted the epidemiological and statistical analyses of the study. PG made a first draft of the paper and all authors made relevant contributions to successive versions. All authors reviewed and approved the final version of the article.

\section{References}

1. World Health Organization (WHO). WHO SAGE pertussis working group. Background paper. SAGE April 2014. Geneva: WHO; 2014. Available from: http://www.who.int/immunization/ sage/meetings/2014/april/1_Pertussis_background_FINAL4_ web.pdf?ua $=$

2. Liu L, Johnson HL, Cousens S, Perin J, Scott S, Lawn JE, et al. Global, regional, and national causes of child mortality: an updated systematic analysis for 2010 with time trends since 2000. Lancet. 2012;379(9832):2151-61. DOI: 10.1016/S01406736(12)60560-1 PMID: 22579125

3. Cherry JD. Epidemic pertussis in 2012--the resurgence of a vaccine-preventable disease. $N$ Engl J Med. 2012;367(9):785-7. DOI: 10.1056/NEJMp1209051 PMID: 22894554

4. Crespo I, Cardeñosa N, Godoy P, Carmona G, Sala MR, Barrabeig I, et al. Epidemiology of pertussis in a country with high vaccination coverage. Vaccine. $2011 ; 29(25): 4244-8$. DOI: 10.1016/j.vaccine.2011.03.065 PMID: 21496465

5. Jenkinson D. Duration of effectiveness of pertussis vaccine: evidence from a 10 year community study.Br Med J (Clin Res Ed). 1988;296(6622):612-4. DOI: 10.1136/bmj.296.6622.612 PMID: 3126927

6. Witt MA, Katz PH, Witt DJ. Unexpectedly limited durability of immunity following acellular pertussis vaccination in preadolescents in a North American outbreak.Clin Infect Dis. 2012;54(12):1730-5. DOI: 10.1093/cid/cis287 PMID: 22423127

7. Cortese MM, Baughman AL, Zhang R, Srivastava PU, Wallace GS. Pertussis hospitalizations among infants in the United States, 1993 to 2004.Pediatrics. 2008;121(3):484-92. DOI: 10.1542/peds.2007-1393 PMID: 18310196

8. Lambert HJ. Epidemiology of a small pertussis outbreak in Kent County, Michigan.Public Health Rep. 1965;80(4):365-9. DOI: 10.2307/4592424 PMID: 14279983

9. Pertussis vaccines: WHO position paper - September 2015 Wkly Epidemiol Rec. 2015;90(35):433-58.PMID: 26320265

10. Gustafsson L, Hessel L, Storsaeter J, Olin P. Long-term follow-up of Swedish children vaccinated with acellular pertussis vaccines at 3, 5, and 12 months of age indicates the need for a booster dose at 5 to 7 years of age.Pediatrics. 2006;118(3):978-84. DOI: 10.1542/peds.2005-2746 PMID: 16950988

11. Klein NP, Bartlett J, Fireman B, Rowhani-Rahbar A, Baxter R. Comparative effectiveness of acellular versus whole-cell pertussis vaccines in teenagers.Pediatrics. 2013;131(6):e1716 22. DOI: $10.1542 /$ peds.2012-3836 PMID: 23690518

12. Klein NP, Bartlett J, Rowhani-Rahbar A, Fireman B, Baxter R. Waning protection after fifth dose of acellular pertussis vaccine in children.N Engl J Med. 2012;367(11):1012-9. DOI: 10.1056/NEJMoa1200850 PMID: 22970945

13. Sizaire V, Garrido-Estepa M, Masa-Calles J, Martinez de Aragon MV. Increase of pertussis incidence in 2010 to 2012 after 12 years of low circulation in Spain.Euro Surveill. 2014;19(32):20875. DOI: 10.2807/1560-7917. ES2014.19.32.20875 PMID: 25139074

14. Bisgard KM, Pascual FB, Ehresmann KR, Miller CA, Cianfrini $C$, Jennings $C E$, et al. Infant pertussis: who was the source? Pediatr Infect Dis J. 2004;23(11):985-9. DOI: 10.1097/01. inf.0000145263.37198.2b PMID: 15545851

15. Wendelboe AM, Njamkepo E, Bourillon A, Floret DD, Gaudelus J, Gerber M, et al. , Infant Pertussis Study Group. Transmission of Bordetella pertussis to young infants.Pediatr Infect Dis J. 2007;26(4):293-9. DOI: 10.1097/01.inf.0000258699.64164.6d PMID: 17414390

16. Kowalzik F, Barbosa AP, Fernandes VR, Carvalho PR, AvilaAguero ML, Goh DYT, et al. Prospective multinational study of pertussis infection in hospitalized infants and their household contacts. Pediatr Infect Dis J. 2007;26(3):238-42. DOI: 10.1097/01.inf.0000256750.07118.ee PMID: 17484221

17. Deen JL, Mink CA, Cherry JD, Christenson PD, Pineda EF, Lewis $\mathrm{K}$, et al. Household contact study of Bordetella pertussis infections. Clin Infect Dis. 1995;21(5):1211-9. DOI: 10.1093/ clinids/21.5.1211 PMID: 8589145

18. Wirsing von König CH, Postels-Multani S, Bock HL, Schmitt HJ. Pertussis in adults: frequency of transmission after household exposure.Lancet. 1995;346(8986):1326-9. DOI: 10.1016/S01406736(95)92343-8 PMID: 7475771

19. Tiwari T, Murphy TV, Moran J, National Immunization Program, CDC. Recommended antimicrobial agents for the treatment and postexposure prophylaxis of pertussis: 2005 CDC Guidelines. MMWR Recomm Rep. 2005;54(RR-14):1-16.PMID: 16340941

20. Instituto Nacional de Estadística (INE). Cifras de población y censos demográficos. [National Statistics Institute. Population figures and Demographic Censuses and Population figures]. 2016 data. Madrid: INE. [Accessed 31 Oct 2016]. Spanish. Available from: http://www.ine.es/inebaseDYN/cp30321/ $\mathrm{cp}$ inicio.htm

21. Baptista PN, Magalhães VS, Rodrigues LC. Children with pertussis inform the investigation of other pertussis cases among contacts.BMC Pediatr. 2007;7(1):21. DOI: 10.1186/1471 2431-7-21 PMID: 17518997

22. European Centre for Disease Prevention and Control (ECDC). Systematic review on the incubation and infectiousness/ shedding period of communicable diseases in children. Stockholm: ECDC; 2016. Available from: http://ecdc.europa.eu/ en/publications/publications/systematic-review-incubationperiod-shedding-children.pdf

23. Roorda L, Buitenwerf J, Ossewaarde JM, van der Zee A. A real-time PCR assay with improved specificity for detection and discrimination of all clinically relevant Bordetella species by the presence and distribution of three Insertion Sequence elements.BMC Res Notes. 2011;4(1):11. DOI: 10.1186/17560500-4-11 PMID: 21255383

24. Resumen anual 2011. [Annual summary 2011]. Boletin Epidemiológico Semanal. 2011;19(1):255. Spanish. Available from: http://revista.isciii.es/index.php/bes/article/ view/689/721

25. Las formas de la convivencia. [Ways of cohabitation. National Statistics Institute.]. Boletin informative del Instituto Nacional de Estadistica. July 2014. [Accessed 31 Oct 2016]. Available 
from: http://www.ine.es/ss/Satellite?L=es_ES\&c=INECifrasINE C\&cid $=1259944407896 \& p=1254735116567 \&$ pagename $=$ Prod uctosYServicios\%2FINECifrasINE_C\%2FPYSDetalleCifrasINE

26. Wirsing von König CH, Postels-Multani S, Bogaerts H, Bock HL, Laukamp S, Kiederle S, et al. Factors influencing the spread of pertussis in households. Eur J Pediatr. 1998;157(5):391-4. DOI: $10.1007 /$ s004310050836 PMID: 9625336

27. de Greeff SC, de Melker HE, Westerhof A, Schellekens JF, Mooi FR van BM, van Boven M. Estimation of household transmission rates of pertussis and the effect of cocooning vaccination strategies on infant pertussis.Epidemiology. 2012;23(6):852-60. DOI: 10.1097/EDE.ob013e31826c2b9e PMID: 23018969

28. Ward JI, Cherry JD, Chang SJ, Partridge S, Lee H, Treanor $J$, et al. Efficacy of an acellular pertussis vaccine among adolescents and adults. N Engl J Med. 2005;353(15):1555-63. DOI: 10.1056/NEJMoa050824 PMID: 16221778

29. Baptista PN, Magalhães V, Rodrigues LC, Rocha MW, Pimentel AM. Pertussis vaccine effectiveness in reducing clinical disease, transmissibility and proportion of cases with a positive culture after household exposure in Brazil. Pediatr Infect Dis J. 2006;25(9):844-6. DOI: 10.1097/01. inf.0000232642.25495.95 PMID: 16940847

30. Baptista PN, Magalhães VS, Rodrigues LC. The role of adults in household outbreaks of pertussis. Int J Infect Dis. 2010;14(2):e111-4. DOI: 10.1016/j.ijid.2009.03.026 PMID: 19559636

31. Deen JL, Mink CA, Cherry JD, Christenson PD, Pineda EF, Lewis $\mathrm{K}$, et al. Household contact study of Bordetella pertussis infections. Clin Infect Dis. 1995;21(5):1211-9. DOI: 10.1093/ clinids/21.5.1211 PMID: 8589145

32. Sala-Farré MR, Arias-Varela C, Recasens-Recasens A, SimóSanahuja M, Muñoz-Almagro C, Pérez-Jové J. Pertussis epidemic despite high levels of vaccination coverage with acellular pertussis vaccine. Enferm Infecc Microbiol Clin. 2015;33(1):27-31. DOI: 10.1016/j.eimc.2013.09.013 PMID: 24216286

33. Moraga F, Roca J, Méndez C, Rodrigo C, Pineda V, Martinez A, et al. , TOSCA Study Group. Epidemiology and surveillance of pertussis among infants in Catalonia, Spain, during 19972001.Pediatr Infect Dis J. 2005;24(6):510-3. DOI: 10.1097/01. inf.0000164701.50766.62 PMID: 15933560

34. Althouse BM, Scarpino SV. Asymptomatic transmission and the resurgence of Bordetella pertussis.BMC Med. 2015;13(1):146. DOI: 10.1186/s12916-015-0382-8 PMID: 26103968

35. Marchant CD, Loughlin AM, Lett SM, Todd CW, Wetterlow LH, Bicchieri R, et al. Pertussis in Massachusetts, 1981-1991: incidence, serologic diagnosis, and vaccine effectiveness. J Infect Dis. 1994;169(6):1297-305. DOI: 10.1093/ infdis/169.6.1297 PMID: 8195607

36. Plans $P$, Álvarez E, de Ory F, Campins M, Payà T, Balfagón P, et al. Prevalence of antibody to Bordetella pertussis in neonates and prevalence of recent pertussis infection in pregnant women in Catalonia (Spain) in 2003 and 2013. Pediatr Infect Dis J. 2014;33(11):1114-8. DOI: 10.1097/INF.0000000000000413 PMID: 24871642

37. McGuiness CB, Hill J, Fonseca E, Hess G, Hitchcock W, Krishnarajah $\mathrm{G}$. The disease burden of pertussis in adults 50 years old and older in the United States: a retrospective study. BMC Infect Dis. 2013;13(1):32. DOI: 10.1186/1471-2334-13-32 PMID: 23343438

38. Iroh Tam PY, Menk JS, Hughes J, Kulasingam SL. An ecological analysis of pertussis disease in Minnesota, 2009-2013. Epidemiol Infect. 2016;144(4):847-55. DOI: 10.1017/ So950268815002046 PMID: 26330135

39. Warfel JM, Zimmerman LI, Merkel TJ. Acellular pertussis vaccines protect against disease but fail to prevent infection and transmission in a nonhuman primate model.Proc Natl Acad Sci USA. 2014;111(2):787-92. DOI: 10.1073/pnas.1314688110 PMID: 24277828

40. van den Biggelaar AH, Poolman JT. Predicting future trends in the burden of pertussis in the 21st century: implications for infant pertussis and the success of maternal immunization.Expert Rev Vaccines. 2016;15(1):69-80. DOI: 10.1586/14760584.2016.1105136 PMID: 26559122

41. Bertilone C, Wallace T, Selvey LA. Finding the 'who' in whooping cough: vaccinated siblings are important pertussis sources in infants 6 months of age and under.Commun Dis Intell Q Rep. 2014;38(3):E195-200.PMID: 25391405

42. Baxter R, Bartlett J, Rowhani-Rahbar A, Fireman B, Klein NP. Effectiveness of pertussis vaccines for adolescents and adults: case-control study.BMJ. 2013;347(jul17 1):f4249. DOI: 10.1136/ bmj.f4249 PMID: 23873919

43. Sheridan SL, Ware RS, Grimwood K, Lambert SB. Unexpectedly limited durability of immunity following acellular pertussis vaccination in preadolescents in a North American outbreak.
Clin Infect Dis. 2012;55(10):1434-5, author reply 1435-6. DOI: 10.1093/cid/cis672 PMID: 22871826

44. Zhang L, Prietsch SO, Axelsson I, Halperin SA, et al. Acellular vaccines for preventing whooping cough in children. Cochrane Database Syst Rev. 2014; (9):CDo01478.PMID: 25228233

45. Dodhia H, Crowcroft NS, Bramley JC, Miller E. UK guidelines for use of erythromycin chemoprophylaxis in persons exposed to pertussis.J Public Health Med. 2002;24(3):200-6. DOI: 10.1093/pubmed/24.3.200 PMID: 12831090

46. Friedman DS, Curtis CR, Schauer SL, Salvi S, Klapholz H, Treadwell T, et al. Surveillance for transmission and antibiotic adverse events among neonates and adults exposed to a healthcare worker with pertussis. Infect Control Hosp Epidemiol. 2004;25(11):967-73. DOI: 10.1086/502328 PMID: 15566032

47. Thampi N, Gurol-Urganci I, Crowcroft NS, Sander B. Pertussis post-exposure prophylaxis among household contacts: a costutility analysis.PLoS One. 2015;10(3):e0119271. DOI: 10.1371/ journal.pone.0119271 PMID: 25747269

\section{License and copyright}

This is an open-access article distributed under the terms of the Creative Commons Attribution (CC BY 4.0) Licence. You may share and adapt the material, but must give appropriate credit to the source, provide a link to the licence, and indicate if changes were made.

This article is copyright of the authors, 2016. 